\title{
FACTORES DE RIESGO ASOCIADOS A MORBIMORTALIDAD PERINATAL PRECOZ EN EL HOSPITAL SAN JUAN DE DIOS. ANCASH, PERÚ
}

\author{
Lidia Mariana Culi-Joyllo ${ }^{1}$
}

1 Médico Pediatra, Hospital San Juan de Dios. Ancash, Perú.

\section{RESUMEN}

Objetivo: Identificar los factores de riesgo asociados a morbimortalidad perinatal precoz del Hospital San Juan de Dios, ubicado en la región Ancash, el año 2010. Material y métodos: Se realizó un estudio retrospectivo, transversal y analítico, la población estuvo conformada de 625 gestantes, se tomó una muestra al azar de 100 gestantes, utilizando una ficha técnica. Para el análisis estadístico se halló el Odds Ratio (OR) y el chi cuadrado a través del programa SPSS versión 14, con intervalo de confianza al 95\%. Resultados: Los recién nacidos con complicaciones fueron 33, el total de recién nacidos muertos fueron 12 por muerte fetal tardía y neonatal precoz de $945 \mathrm{~g}$, a más y los controles 88 nacidos vivos, seleccionados al azar. La tasa de mortalidad precoz fue 12\%. Los factores de riesgo fueron: Edad Materna 35 años a más (OR=4,54), Multiparidad $(\mathrm{OR}=3,77)$, Control prenatal ausente o inadecuado $(\mathrm{OR}=3,87)$, Inicio de parto inducido o cesárea electiva $(\mathrm{OR}=21,5)$, Trabajo de parto precipitado o prolongado $(\mathrm{OR}=10,5)$, Depresión $(\mathrm{OR}=37,40)$ Prematuridad $(\mathrm{OR}=9,67)$. La presencia de depresión al nacer, inicio de parto inducido, trabajo de parto prolongado, bajo peso y prematuridad, tienen altos valores predictivos. Conclusiones: Los factores de riesgo del recién nacido tienen mayor asociación y alto valor predictivo para mortalidad perinatal precoz en el hospital San Juan de Dios de Ancash, Perú.

Palabras claves: Mortalidad perinatal, prematuridad, factores de riesgo, Perú.(fuente: DeCS BIREME)

\section{RISK FACTORS ASSOCIATED WITH PERINATAL MORBIDITY EARLY IN THE SAN JUAN DE DIOS HOSPITAL. ANCASH, PERU}

\begin{abstract}
Objective:Toldentify the risk factors associated with perinatal morbidity of the early San Juan de Dios Hospital, located in the Ancash region, 2010. Material and methods:It was realized a retrospective study, analytical cross the population consisted of 625 pregnant women, it took a random sample of 100 pregnant women, using a technical sheet. For the statistical analysis found the Odds Ratio (OR) and the chi-square through the program SPSS version 14, with 95\% confidence interval.Results: Newborns with complications were 33, the total number of newborns killed were 12 by death late fetal and early neonatal of $945 \mathrm{~g}$, and the controls 88 live births, selected at random. The early mortality rate was $12 \%$. The risk factors were: maternal age 35 years to more $(\mathrm{OR}=4.54)$, multiparity $(\mathrm{OR}=3.77)$, prenatal care absent or inadequate $(\mathrm{OR}=3.87)$, marking the beginning of induced labor or elective cesarean section (OR=21.5 ), work of premature birth or prolonged (OR=10.5 ), depression $(\mathrm{OR}=37.40$ ) Prematurity $(\mathrm{OR}=9.67)$. The presence of depression at birth, initiation of induced labor, prolonged labor, low birth weight and prematurity, have high predictive values. Conclusions: The risk factors of the newborn have greater partnership and high predictive value for early perinatal mortality in the San Juan de Dios hospital in Ancash, Peru.
\end{abstract}

Key words: Perinatal mortality, prematurity, risk factors, Peru. (source: MeSH NLM)

\section{INTRODUCCIÓN}

El hospital San Juan de Dios funcionaba como centro de salud y después de tomar posesión de un terreno donado, construido y equipado adecuadamente, inicia su funcionamiento como hospital San Juan de Dios incorporándose al Ministerio de Salud (MINSA) con 37 años de labor institucional en las jurisdicciones de las provincias de Huaylas, Yungay y Corongo, la región Ancash en Perú.

Los factores de riesgo de morbimortalidad perinatal precoz adquieren importancia al proporcionarnos una imagen del nivel de desarrollo y calidad de salud de los pueblos, con influencia en la calidad de vida del recién nacido, desarrollo físico, neurológico y mental condicionando su futuro.
La Organización Mundial de la Salud (OMS) informa que en el Perú la mortalidad ligada al embarazo y parto representa el $56 \%$, y estima que en el mundo nacen muertos alrededor de 4,3 millones de niños y 7,6 millones de muertes perinatales, el $98 \%$ ocurren en países en vías de desarrollo, considerándose un problema prioritario de salud pública a estudiar ${ }^{(1,2)}$.

Los factores de riesgo más frecuentes como la ausencia de control prenatal permanente, la edad materna, estado civil, multiparidad, el trabajo de parto prolongado, el trabajo de parto inducido, así como los problemas geográficos, económicos y culturales agregadas a las fallas estructurales del sistema de salud, corresponden a los altos índices de depresión, bajo peso al nacer, prematuridad, mortalidad perinatal en el recién nacido contribuyendo con el estudio a 
la reducción de causas evitables en la atención de la madre y del recién nacido menor de siete días.

La mayoría de los daños obstétricos y los riesgos para la salud de la madre y del niño pueden ser prevenidos, detectados y tratados con éxito mediante la realización de actividades eminentemente preventivas y la eliminación o racionalización de algunas prácticas que llevadas a cabo en forma rutinaria aumenta los riesgos, por lo que es importante conocer el fenómeno en la entidad con el siguiente objetivo: Identificar los factores de riesgos asociados a morbimortalidad perinatal precoz Hospital San Juan de Dios.

Sobre estos aspectos se detallan en el presente estudio para contribuir con la expectativa de vida y la calidad de vida del recién nacido con un seguimiento adecuado que no termina cuando recibe alta médica de la Unidad Neonatal correspondiente $^{(3,4)}$.

Las características de las madres que se atienden son de recursos precarios y escaso control prenatal de los cuales se midió a través de la ficha técnica considerando los antecedentes generales de las gestantes, los factores de riesgo obstétrico y las complicaciones durante la gestación y el parto. El análisis estadístico se realizó a través de Microsoft Excel versión 2007, y SPPS versión 14.

\section{MATERIAL Y MÉTODOS}

El método utilizado es deductivo de tipo analítico transversal, utilizando el muestreo probabilístico aleatorio simple. Se consideró 100 pacientes que realizaron parto en el hospital San Juan de Dios de Caraz; elaborándose una ficha técnica probada a través de una muestra piloto para la calidad de los datos obtenidos. Se validó el instrumento a través de Odds Ratio y Chi cuadrado con intervalo de confianza al $95 \%$.

\section{RESULTADOS}

Durante el período de Enero a Diciembre del 2010, se atendieron 625 partos en el Hospital San Juan de Dios de Caraz-Ancash, del estudio realizado se encontró factores maternos asociados: el $44 \%$ son mujeres mayores de 35 años, el $64 \%$ convivientes, $19 \%$ tuvo más de 5 partos, el $62 \%$ no acudió a los controles prenatales, el $8 \%$ tuvo trabajo de parto prolongado, el $6 \%$ de mujeres tuvieron trabajo de parto inducido.

Se evidencio prematuridad en un $40 \%$, mientras que la mortalidad perinatal se dio en un $12 \%$.Un $39 \%$ de total, fue considerado como recién nacido de bajo peso.

Los factores de riesgo asociados al recién nacido: el $40 \%$ fueron recién nacidos pre términos, $31 \%$ presentan depresión, $39 \%$ tienen bajo peso al nacer, el $12 \%$ corresponden a las muertes del recién nacido ${ }^{(6)}$.

\section{DISCUSIÓN}

El Centro Latinoamericano de Perinatología (CLAP) realizó un estudio de morbimortalidad perinatal, donde indica que el Perú cuenta con 3 regiones geográficas, la región Sierra por encima de los $3000 \mathrm{msnm}$, tiene la mayor tasa 32.3/ 1000 nacidos vivos, el riesgo de morir es de 1.8 veces mayor que la costa.

De los factores maternos corresponden: el $56 \%$ de mujeres resultaron con una edad mayor a 35 años, el $64 \%$ resultaron con estado civil de conviviente que incluso están asociadas al factor riesgo de la multiparidad (19\%), caracterizando a este grupo las labores cotidianas que realiza y atención del parto, por limitaciones geográficas, económicas o culturales ( $16,17,18)$. Revisados con otros estudios se confirma que son factores de riesgo perinatal que corresponden a un grupo de riesgo para muerte perinatal, la edad en sí es asociación con la gravedad de complicaciones que se presentan durante el embarazo tales como diabetes, preeclamsia, amenaza de parto pretermino, recién nacidos de bajo peso, asfixia prenatal ${ }^{(13)}$.

El factor de riesgo del control prenatal inadecuado puede generar complicaciones durante el embarazo y atención del parto como la preeclampsia, hemorragia del tercer trimestre y retardo del crecimiento intrauterino. La gran multiparidad y el período intergenésico corto, son factores con impacto en la morbimortalidad precoz. Luke y Brown ${ }^{(14)}$, mostraron que el control prenatal es un factor protector para recién nacido.

El factor de riesgo de trabajo de parto prolongado (8\%) conduce al sufrimiento fetal provocado por una duración mayor de 9 horas de trabajo de parto.

De los factores de riesgo del recién nacido de nuestro estudio son los prematuros (40\%), uno de los factores que guarda estrecha relación con el peso del recién nacido (39\%), con depresión (31\%) que tienen mayor probabilidad de morir en etapa perinatal y que los autores aclaran que en los países en vías de desarrollo que la primera causa de muerte perinatal es la prematuridad seguida por el peso del recién nacido ${ }^{(10,11)}$.

Como conclusión final mencionamos que la tasa de mortalidad neonatal fue del $12 \%$, los factores de riesgo de la madre asociado con los fallecidos menores de 7 días en el Hospital San Juan de Dios de Caraz fueron la edad, multiparidad, la falta de control prenatal adecuada.

La información obtenida en este trabajo es una aproximación al problema para conocer la incidencia, la importancia de los factores asociados y el nivel de cambio esperado en la muerte neonatal. La diferencia de los resultados se explica por ser un hospital referencial con embarazos de alto riesgo obstétrico, con cuadros de amenaza de parto prematuro, hipertensión del embarazo, entre otros. 
Tabla 1. Factores de riesgo asociados a morbimortalidad perinatal precoz

\begin{tabular}{llcc}
\hline \multicolumn{1}{c}{ Factores } & \multicolumn{1}{c}{ Rango } & OR & IC \\
\hline Preconcepcionales & $<5$ o $>5$ hijos & 3,776 & $1,048-13,606$ \\
Paridad & $<35$ o $>35$ años & 4,543 & $1,149-17,960$ \\
Edad materna & Sin CPN/con CPN & 3,867 & $1,077-13,888$ \\
Del embarazo & Normal & 10,500 & $2,197-50,177$ \\
Control prenatal (CPN) & Inducido/espontaneo & 21,500 & $3,396-136,125$ \\
Del parto & & & \\
Duración del parto & Con/sin depresión & 37,400 & $4,548-307,545$ \\
Inicio del parto & $<37$ o $>37$ semanas & 9,667 & $1,989-46,947$ \\
Del recién nacido & $<2500$ o $>2500 \mathrm{~g}$ & 10,172 & $2,091-49,481$ \\
Depresión & & & \\
Prematuridad & & & \\
Peso al nacer & &
\end{tabular}

Fuentes de financiamiento: autofinanciado.

Conflicto de intereses: el autor declara no tener conflictos de interés.

Correspondencia: Lidia Mariana Culi Joyllo

Correo electrónico: lidia_culi@hotmail.com

\section{REFERENCIAS BIBLIOGRÁFICAS}

1. Chalmers B, Mangiaterra V, Porter R. WHO principles of perinatal care: the essential antenatal, perinatal, and postpartum care course. Birth. 2001; 28(3): 202-7.

2. Hobar JD, Badger GJ, Carpenter JH, et al. Trends in mortality and morbidity for very low birth weight infants, 1991 to 1999. Pediatrics 2002;110(1):143151.

3. Ticona M, Huanco D. Mortalidad Perinatal Hospitalaria en el Perú: Factores de Riesgo. Rev. Chil. Obstetricia Ginecologica 2005; 70(5): 313-317

4. Organización Panamericana de la Salud. Guías para la vigilancia de la mortalidad materna. Washington, D.C. 1996.

5. Tejada R. Mortalidad materna y perinatal en el Distrito de Yauli-Huancavelica 1997. Perú: Ginecología y Obstetricia 1999; 45 (2):131-6.

6. Ticona $\mathbf{M}$. Factores de riesgo asociados a mortalidad perinatal. Experiencia en el Hospital Hipólito Unanue de Tacna. 1992-1997. Rev. Peruana Ginecología y Obstetricia 1998;44(3): 19927

7. Organización Mundial de la Salud. Situación de Salud de las Américas. Indicadores Básicos 2001 OPS/OMS. Ginebra, 2001.

8. Ticona M, Huanco D. Factores de riesgo de la mortalidad perinatal en el Perú. Rev Per Ginecol Ostet. 2003; 49(4): 227-236.

9. Diaz L, Huaman M, Necochea I et al.Mortalidad perinatal en el Hospital Nacional Edgardo Rebagliati Martins.Rev Med Hered. 2003; 14(3):117-121.

10. Vásquez JC, Vásquez $C$, Rodríguez $\mathbf{P}$. Epidemiología de la rotura prematura de membranas en un hospital ginecoobstétrico. Rev. Cubana Obstet. Ginecol. 2003; 29 (2):3-7.
11. Palomino W, Murillo J. Incidencia de mortalidad Perinatal y Factores Asociados a Mortalidad Neonatal Precoz en el Hospital Daniel Alcides Carrión del Callao. Anales de la Facultad de Medicina 1994; 58(2): 92-8.

12. González G. Patrones Demográficos, Reproductivos y de morbimortalidad en las poblaciones de altura del Perú. Acta Andina 1998; 7(2): 85-93.

13. Ticona $\mathbf{M}$, Huanco $\mathbf{D}$. Factores de riesgo de la mortalidad perinatal en hospitales del Ministerio de Salud del Perú. Rev Cubana Obstet Ginecol. 2012; 37(3): 432-443.

14. Instituto Nacional de Estadística e Informática. Encuesta Nacional de Demografía y Salud 2000. Lima, 2001.

15. Díaz JV, Hernández J, Crespo J, Deulofeo $\mathbf{M}$. Embarazo post-término. Seguimiento paraclínico, correlación de variables. Rev Cubana Obstet Ginecol 1997; 23(1):37-42.

16. Hernández JH, Hernández D, Garcia $L$ et al. Resultados perinatales y maternos en los embarazos en edad madura. Rev Cubana Obstet Ginecol. 2003; 29(2):28-9.

17. Centro Latinoamericano de Perinatología y Desarrollo Humano. Peso al nacer y morbimortalidad perinatal. Documento Interno del CLAP Nº 3/86. Uruguay. 1986.

18. Manuel T, Huanco D. Factores de riesgo de la mortalidad perinatal en hospitales del ministerio de salud del Perú. Rev chil obstet Gineco. 2005; 70(5):313-317.

19. Silvia H. Impacto del perfil biofísico fetal sobre la morbilidad y mortalidad perinatal en embarazos de alto riesgo. [Tesis para obtener el grado de Maestro en Ciencias Médicas] México: Universidad de COLIMA. 2000. 57 pp.

20. Castellanos JC, Rodríguez H.Factores relacionados con mortalidad por sepsis neonatal en el hospital Dr. Adolfo Prince Lara. Puerto Cabello. 
Venezuela. Enero - Octubre 2009. Revista electrónica portales medicos.com [online] Noviembre 2010 [fecha de acceso 25 de Marzo del 2011] URL disponible

en: http://www.portalesmedicos.com/publicaciones/articl es/2271/1/Factores-relacionados-con-mortalidadpor-sepsis-neonatal

21. Cabero L et al. Obstetricia y medicina materno-fetal. obstétrico. 1era ed. España. Editorial Panamericana, 2007.

22. Hernández JM. Protocolos asistenciales en Obstetricia y Ginecología. Tomo I. Obstetricia. Sego. Madrid 1993.

23. Avery G. Neonatología: Fisiopatología y manejo del recién nacido. Buenos aires. $3^{\circ}$ edición. Editorial Panamericana. 1992.

24. Caldeyro R. Mortalidad perinatal y nivel socioeconómico. Rev. Ciencia Hoy. 1991; 2(12): 504.

25. Centro Latinoamericano de Perinatología y Desarrollo Humano. Situación materna y perinatal de la Región. Boletín Salud Perinatal. Montevideo. 1998; 6(16): 64.

26. Fabré E. Manual de asistencia al parto normal. Talleres gráficos de editorial Luis Vives. Zaragoza. 1993.

27. Saavedra D, Valdés S, Bardales J, Essien J,de la Torre Y. Morbimortalidad perinatal de la rotura prematura de membrana en el embarazo pretérmino. Clínica e Investigación en Ginecología y Obstetricia. 2006; 33(3):102-6.

28. De la Torre J, Salvador J. Factores de riesgo asociados a muerte neonatal en el Hospital Cayetano Heredia 1992-1993. Rev Ginecol Obstet 1995; 45(1): 83-7.

29. Mandujano $\mathbf{M}$, Sánchez $\mathbf{M}$. La morbimortalidad perinatal y la génesis del daño neurológico. Rev. Cienc. Clín 2005; 6(2): 79 - 90.

30. Laffita A., Ariosa J. Comportamiento de la mortalidad perinatal en el hospital "América Arias" en un período de 3 años. Rev. Cubana Obstet Ginecol. 2004; 30(3):8-15.

31. González G. Patrones Demográficos, Reproductivos y de morbimortalidad en las poblaciones de altura del Perú. Acta Andina 1998; 8(1): 85-93.

32. Cervantes R. Muerte materna y muerte perinatal en los Hospitales del Perú. Ministerio de Salud, Sociedad Peruana de Obstetricia y Ginecología, OPS. Lima 1988.

33. Salazar M. La edad materna avanzada como factor de riesgo de mortalidad materna y perinatal. Perú: Ginecol Obst 1999; 45(2): 124-30.

34. Osorio-Amézquita C, Romero-Vázquez A. Factores de riesgo asociados a mortalidad neonatal precoz. Salud en Tabasco. 2008; 14(1-2): 721-726.

Recibido: 12 / 10 / 2012

Aceptado para publicación: 23 / 12 / 2012 